\title{
ZUR DATIERUNG VON HEINRICH VON KLEISTS POLITISCHEN SCHRIFTEN DES JAHRES 1809
}

Kleists politische Schriften werden bekanntlich weder in den wenigen uns erhaltenen Briefen vom Jahre 1809 noch in den bisher bekannt gewordenen Zeugnissen aus seiner Umgebung einzeln erwähnt, was dazu beigetragen hat, daß die Forschung über "mehr oder weniger vage Datierungsversuche"' nicht hinausgekommen ist. Die genauere zeitliche Einordnung wird auch dadurch erschwert, daß einige dieser Prosastücke keine Anhaltspunkte zur Datierung aufzuweisen scheinen. Hier soll der Versuch unternommen werden, durch Einbeziehung bisher unbeachteter Quellen mehrere Texte dieser Gruppe etwas präziser zu datieren.

Zunächst erhebt sich die Frage, welche Schriften noch vor Kleists Abreise aus Dresden am 29. April 1809 entstanden sein könnten. Der 1808 beginnende und sich 1809 verstärkende Aufbau des österreichischen Propagandawesens wird auf antinapoleonisch eingestellte Autoren wie Kleist einen starken Anreiz ausgeübt haben. ${ }^{2} \mathrm{Daß}$ er sich schon im Frühjahr 1809 agitatorisch betätigte, erhellt daraus, daß er Collin sowohl die Hermannsschlacht ${ }^{3}$ wie auch patriotische Gedichte zur Verfügung stellte und überdies - vielleicht beeindruckt vom Erfolg der Lieder österreichischer Wehrmänner Collins - eine Sammlung seiner politischen Lyrik plante. ${ }^{4}$ Möglicherweise hat er damảls bereits Ähnliches auf dem Gebiet der Prosa versucht.

Es ist nicht auszuschließen, daß das Lehrbuch der französischen Journalistik schon in Dresden niedergeschrieben wurde, und zwar nicht erst kurz vor Kleists Abreise. Die darin enthaltene Kritik an der französischen Kriegsberichterstattung bezieht sich eventuell auf die Einstellung der französischen Presse zum spanischen Unabhängigkeitskrieg. Besonders im Zeitraum Sommer 1808 bis Frühjahr 1809 war nämlich der Vergleich französischer und anderer Nachrichtenquellen über die Vorgänge in Spanien ein Hauptthema der politischen Diskussion in den deutschen Staaten.

Bei zwei anderen Schriften Kleists, die im Gegensatz zum Lehrbuch der französischen Journalistik datierbare Anspielungen enthalten, läßt sich mit größerer Sicherheit annehmen, daß sie vielleicht noch in Dresden verfaßt wurden. Dem im Brief eines politischen Pescherä verwendeten Artikel aus dem Nürnberger Korrespondenten von und für Deutschland ${ }^{6}$ vom 25. April 1809 kann Kleist noch kurz vor seiner Abreise zu Gesicht bekommen haben, selbst wenn man die Postwege einrechnet. Die in der Nachschrift zum Brief eines rheinbündischen Offiziers erwähnte Überbringung der Nachricht vom Sieg Napoleons bei Landshut am 21. April 1809 durch Montesquiou fand tatsächlich statt, was einen etwas genaueren Terminus post quem als den bisher angenommenen ermöglicht. Kleist wird unmittelbar vor seiner Abreise davon gehört haben, daß Montesquiou dem sächsis- 
chen König die Siegesnachricht am Abend des 25. April in Leipzig überbrachte. $^{7}$

Bisher ist noch nicht gründlich untersucht worden, ob nicht auch das kleine Prosastück Die Bedingung des Gärtners eine datierbare Anspielung enthält. Auszugehen wäre von der herausfordernden abschließenden Frage "Landwehren von Österreich! Warum wollt ihr bloß, innerhalb eures Landes, fechten?"8 Dem kaiserlichen Patent zur Errichtung der Landwehr vom 9. Juni 1808 zufolge sollten diese Truppen nämlich nur innerhalb der Landesgrenzen eingesetzt werden, aber in den Monaten vor dem Kriegsausbruch (9. April 1809) begann die österreichische Führung auf ihren Einsatz außerhalb Österreichs hinzuarbeiten. Richard Samuel erwähnt in seiner Dissertation die Möglichkeit, daß Die Bedingung des Gärtners schon in Dresden entstanden sein könnte, ${ }^{9}$ verweist aber zur Begründung lediglich auf einen in der Wiener Zeitung vom 24. März 1809 veröffentlichten Aufruf des Erzherzogs Karl an die Landwehr, worin sie aufgefordert wird, auch jenseits der Grenzen zu dienen. ${ }^{10}$ Dem ließe sich noch hinzufügen, daß am 10. April 1809 tatsächlich Landwehrabteilungen nach Bayern und vom Böhmen her in die Oberpfalz einmarschierten. ${ }^{11}$ Kleists ungeduldige Frage an die Landwehr wird aber erst verständlich, wenn man sie auf den Widerstand bezieht, der sich bei manchen Landwehreinheiten gegen den Einsatz außerhalb Österreichs erhoben hatte. Er könnte noch vor seiner Abreise aus Dresden erfehren haben, daß sich zwei böhmische Landwehrbataillone etwa Mitte April geweigert hatten, die Grenze zu überschreiten. ${ }^{12}$ Diese Ereignisse, welche natürlich von der Presse im französischen Machtbereich aufgegriffen wurden, ${ }^{13}$ müßten Kleist irritiert haben, dessen Auffassung der Funktion der Landwehr seiner Befürwortung eines totalen Endkampfes gegen Napoleon entspringt. Wenn man die Möglichkeit einräumt, daß der den Kriegsverlauf genau beobachtende Dichter auf diese Befehlsverweigerung anspielt, ergäbe sich zunächst also etwa Ende April 1809 als Terminus post quem für die Abfassung der Bedingung des Gärtners.

Die folgenden Überlegungen führen allerdings zu der Vermutung, daß dieser Text erst einige Wochen später, nämlich etwa Anfang Juni entstand. Der Sieg bei Aspern (21. und 22. Mai 1809), ermöglichte es der österreichischen Führung, wieder Operationen jenseits der Grenzen vorzubereiten. Da für die Landwehrtruppen keine rechtliche Verpflichtung zum Dienst im Ausland bestand, mußte sie dazu überredet werden. Hierbei spielte die österreichische Propaganda, der sich bekanntlich auch Kleist zur Verfügung stellte, eine wichtige Rolle. Am 31. Mai 1809, dem Tag der Rückkehr Kleists nach Prag, erließen Joseph Graf von Wallis, Oberstburggraf und seit dem 24. April 1809 Generallandeskommissär, und Johann Graf von Riesch, der kommandierende General in Böhmen, in Prag zur Siegesfeier einen Aufruf an die böhmische Landwehr, den Kleist höchstwahrscheinlich zu Gesicht bekam. Nach Hervorhebung der Verdienste böhmischer Landwehreinheiten in der Schlacht bei Aspern wird den Trup- 
pen weiterer Ruhm in Aussicht gestellt, ${ }^{14}$

wenn ihr dem Rufe des Vaterlandes folgt, euch auch außer den Gränzen zu treten, bereitwillig erkläret, und den Feind überall anzugreifen, und zu verfolgen euch anheischig macht ...

Auf denn brave Landwehrmänner! Bedeckt euch nicht bloß in den Erbstaaten, sondern auch außer denselben, auf dem feindlichen Gebiete mit Ruhm. Eurer wartet auf demselben Ehre, Glück und die schönsten Lorbeeren.

Diese Proklamation sowie wahrscheinlich auch Die Bedingung des Gärtners hängen mit den Vorbereitungen zu dem am 10. Juni 1809 beginnenden Einmarsch ins Bayreuthische und nach Sachsen zusammen, an dem sich auch Landwehreinheiten beteiligten. Falls diese Deutung zutrifft, war Die Bedingung des Gärtners für die Germania bestimmt, deren Gründung gerade in jenen Tagen in Prag versucht wurde.

Die Forschung hat bisher oft angenommen, daß auch die Arbeit an Katechismus der Deutschen schon in Dresden begonnen haben könnte. Dieser Auffassung zufolge stellt Kleists angebliche Anspielung auf die Schlacht bei Regensburg vom 23. April 1809 den Terminus post quem dar. ${ }^{15}$ Wenn Kleist im 6. Kapitel des Katechismus davon spricht, daß "Erzherzog Karl die Franzosen, da sie bei Regensburg standen, angegriffen hat," 16 bezieht er sich nicht auf die österreichische Niederlage bei Regensburg vom 23. April, sondern auf die erste größere Schlacht des Krieges, nämlich den am 18. und 19. April 1809 stattfindenden Vorstoß Erzherzog Karls gegen den bei Regensburg aufgestellten Davout. ${ }^{17}$ Aber selbst dieses korrigierte Datum darf höchstwahrscheinlich nicht als Terminus post quem angesehen werden. Bisher hat meistens man stillschweigend vorausgesetzt, daß der Dichter seine Quelle, den von Vitali übersetzten Bürger-Katechismus, schon Ende April 1809 kannte. Johannes Bethke entdeckte zwar diese Vorlage Kleists, ${ }^{18}$ ging aber der Frage nicht nach, wann das 4. und zugleich letzte Heft der der in Wien von Anton Strauß verlegten Sammlung der Aktenstücke über die spanische Thronveränderung, worin der BürgerKatechismus unter Nr. 20 erscheint, veröffentlicht wurde. Aus einem Schreiben des Verlegers vom 28. März 1809 an Franz von Hager, den Leiter der Polizeihofstelle, geht hervor, daß das 3. Heft etwa Ende März auf den Markt kommen sollte und das 4. fast fertig sei. ${ }^{19}$ Vielleicht lag Strauß aus geschäftlichen Gründen ${ }^{20}$ daran, beim Hof den Eindruck zu erwecken, als stünde die Veröffentlichung des 4. Heftes unmittelbar bevor. Es scheint in Vergessenheit geraten zu sein, daß schon Richard Samuel unter Hinweis auf einen Brief Friedrich Schlegels an Hormayr vom 23. Juni 1809 zu dem Schluß gelangte, daß Kleist die Vorlage seines Katechismus wahrscheinlich erst nach seiner Abreise aus Dresdèn zu Gesicht bekam. ${ }^{21}$ Schlegel berichtet nämlich, daß Strauß wegen der am 13. April erfolgen Besetzung Wiens nach Ungarn geflohen sei und fährt dann fort: "Wenn er auch seinen Vorrath von Exemplaren gerettet hat, so sagen Sie ihm, er solle Vorräthe vom 4ten Theil der spanischen Aktenstücke nach Brünn und Prag senden, soviel er kann; auch für das Ausland. Dieser 4te Band ist noch nicht sehr herumgekommen, weil er mitten in die Zeit des Krieges fiel."22 Schlegels 
Aussage gewinnt dadurch an Gewicht, daß er selbst einige der für das 4 . Heft bestimmte Stücke übersetzt hatte und als Sekretär in dem von Friedrich von Stadion geleiteten Oberhofskommissariat über die österreichische Propagandakampagne gut unterrichtet war. ${ }^{23}$

Kleists Vorlage ist also sicher nach Kriegsbeginn, aber höchstwahrscheinlich noch vor der Belagerung Wiens erschienen. Zwei Begleitschreiben zu umfangreichen Lieferungen verschiedener Flugschriften an Friedrich von Stadion, welche der Leiter der Staatskanzlei, Joseph von Hudelist, am 17. und 19. April 1809 in Wien verfaßte, bieten möglicherweise einen Anhaltspunkt für eine genauere Datierung. Während in beiden Briefen Pakete angekündigt werden, die nur die ersten drei Hefte der Sammlung der Aktenstücke enthalten, heißtes im zweiten Brief anschließend: "Item Cent Exemplaires incomplets, c'est a dire, à deux Volumes chacun, parceque le courier n'a pas pu se charger de deux autres Volumes, qui suivront incessement." ${ }^{24}$ Um den 19. April 1809 wird also das 4. Heft erschienen sein, welches wohl infolge des in den darauffolgenden Tagen einsetzenden österreichischen Rückzugs zunächst nur eine geringe Verbreitung fand. Vermutlich hat Kleist es nicht mehr in Dresden lesen können, denn die Postverbindungen zwischen Österreich und Sachsen waren nach Kriegsausbruch zeitweilig unterbrochen worden. ${ }^{25}$ Der Katechismus der Deutschen ist wahrscheinlich frühestens Mitte Mai entstanden. Es ist nämlich kein Zufall, daß Kleist nationale Fragen am Beispiel Sachsens illustriert. In Znaim wird er von den erneuten Diskussionen der österreichischen Führung über eine Invasion Sachsens gehört haben. ${ }^{26}$ Es kann jetzt als gesichert gelten, $\mathrm{da} ß$ er dort von Buols Bemühungen erfuhr, in Sachsen eingesetzt zu werden, womit sich für Kleist die Möglichkeit einer Anstellung als politischer Publizist zu eröffnen schien. ${ }^{27}$

Unter den hier noch nicht erwähnten Schriften, die Kleist wahrscheinlich alle in Böhmen verfaßte, kann man zwei mit einiger Genauigkeit datieren. Die Einleitung zur Germania ist vermutlich etwa Mitte Juni 1809 entstanden, zu einer Zeit also, als sich Kolowrat und Wallis für Kleists Plan einsetzten. Die Entwürfe Über die Rettung von Österreich wird Kleist etwa Anfang September 1809 niedergeschrieben haben. Sie nehmen eine Sonderstellung unter den Schriften ein, denn die scharfe Überwachung der Presse seitens der österreichischen Behörden läßt es als unwahrscheinlich erscheinen, daß sie zur Veröffentlichung in der Germania oder einem anderen Blatt bestimmt waren. ${ }^{28} \mathrm{Im}$ pathetischen Ton der beiden verbleibenden Stücke, Zeitgenossen und Was gilt es in diesem Kriege?, scheint das schockierende Erlebnis der schweren österreichischen Niederlagen bald nach Beginn des Krieges noch nachzuwirken. Kleist Schreckenvisionen eines Untergangs von Staat und Kultur sowie seine Forderung nach totalem Einsatz beziehen hieraus ihre Dringlichkeit. Die in Was gilt es in diesem Kriege? hämmernd wiederholte Wendung "es gilt" ermöglicht vielleicht eine annähernde Datierung auf Mai oder Juni 1809, denn im Brief an Buol vom 25. Mai 1809 verwendet er sie in einem ähnlichen Kontext. 
Hier äußert er nämlich die Hoffnung, daß nach dem Sieg bei Aspern "ein Krieg entsteht, wie er der großen Sache, die es gilt," würdig ist. ${ }^{29}$ Kleists Vergleich des Kabinettkrieges mit einem Schachspiel ${ }^{30}$ unterstützt diese Datierung. Bekanntlich spielten Dahlmann, Kleist und Pfuel etwa Mitte Mai in Znaim das Kriegsspiel, welches, um 1780 aus dem Schachspiel abgeleitet, damals gerade von Pfuel verbessert worden war. ${ }^{31}$

Kleist erwähnt seine politischen Schriften nur einmal, und zwar im Brief an Ulrike vom 17. Juli 1809, in dem er sich an die "schöne Zeit" nach Aspern erinnert: "ich fand Gelegenheit, einige Aufsätze, die ich für ein patriotisches Wochenblatt bestimmt hatte, im Hause des Grf. v. Kollowrat, vorzulesen." ${ }^{32}$ Bereits Rudolf Köpke, der 1862 erstmals die meisten politischen Schriften des Jahres 1809 veröffentlichte, bezog sie auf Grund dieser Briefstelle zu ausschließlich auf das Germania-Projekt, ${ }^{33}$ obwohl Kleists kurzer Rückblick vermutlich die Entstehungsgeschichte dieser Textgruppe stark vereinfacht. Darin ist die Forschung Köpke bis heute nachgefolgt. ${ }^{34}$ Es kann nicht bestritten werden, daß Kleist bald nach seiner Rückkehr in die böhmische Hauptstadt (31. Mai 1809) begann, die meisten oder alle der uns überlieferten Schriften als mögliche Beiträge für die Germania zu betrachten, natürlich mit Ausnahme der später verfaßten Entwürfe Über die Rettung von Österreich. Andererseits besteht die Möglichkeit, daß die bis Mai 1809 entstandenen Schriften ursprünglich nicht im Hinblick auf die Germania konzipiert wurden, darunter auch der kürzlich vom Verfasser entdeckte und demnächst zu veröffentlichende Aufsatz Über die Abreise des Königs von Sachsen aus Dreßden. Diese Texte sollten evtl. anderswo publiziert werden, wobei nicht nur an Zeitungen oder Zeitschriften zu denken wäre. Im Frühjahr 1809 erschien eine Unzahl von heute z.T. verschollenen Flugblättern und Flugschriften jeglichen Umfangs und aller nur denkbaren Stilarten. ${ }^{35}$ Die wahrscheinlich relativ früh entstandenen satirischen Briefe z.B. stellen möglicherweise Teile einer ursprünglich geplanten Sammlung solcher Prosastücke dar. Auch das Lehrbuch der französischen Journalistik und der Katechismus der Deutschen hätten sich im Rahmen der damaligen österreichischen Propagandakampagne durchaus zu einer Einzelveröffentlichung geeignet.

University of Michigan, Ann Arbor

HERMANN F. WEISS

Anmerkungen

1. Rudolf Berg, "Intention und Rezeption von Kleists politischen Schriften des Jahres 1809." In: Text und Kontext. Quellen und Aufsätze zur Rezeptionsgeschichte der Werke Heinrich von Kleists, hrsg. v. Klaus Kanzog, Berlin 1979, S. 196.

2. Vgl. vom Verf. "Heinrich von Kleists politisches Wirken in den Jahren 1808 und 1809. Mit der Edition einer neuentdeckten Origimalhandschrift von 'Was gilt es in diesem Kriege?'." In: Jahrb. d. dt. Schillergesellschaft 25, 1981, S. $9-40$.

3. II, S. 82lf; Brief vom 22. Februar 1809 (römisch/arabische Ziffer für Band/Seite in: 
Heinrich von Kleist. Sämtliche Werke und Briefe, 2 Bde., hrsg. v. H. Sembdner, München 1977).

4. II, S. 824; Brief vom 20. April 1809.

5. Vgl. Rainer Wohlfeil, Spanien und die deutsche Erhebung 1808-1814, Wiesbaden 1965 , S. 102 ff. Siehe auch Johannes Bethke (H. v. Kleist und Österreich, Diss. (masch.) Wien 1932, S. 13f) zu Hinweisen auf Flugschriften über die Kämpfe in Spanien im Dresdener Anzeiger 1808. - Richard Samuel vertritt dagegen in seiner Dissertation die Ansicht, daß Kleist im Lehrbuch der französischen Jounalistik auf die irreführende französische Berichterstattung über Aspern reagiert (Heinrich von Kleist's Participation in the Political Movements of the Years 1805 to 18009, Diss. Cambridge 1938, S. 405).

6. Vgl. II, S. 936f. - Wohlfeil zufolge stellte diese Zeitung übrigens des öfteren ihre Unabhängigkeit gegenüber der französischen Politik unter Beweis (ebd. S. 122-125).

7. Im Bericht des württembergischen Legationssekretärs Carl Heinrich Rheinwald vom 26. April 1809 aus Leipzig verlautet nämlich über diese Nachricht: "sie kam gestem abend durch den vom Schlachrfeld an den König von Sachsen hieher geschickten französischen Ordonnanzoffizier Montesquiou an ..." (Hauptstaatsarchiv Stuttgart, Bestand Württemberg. Gesandtschaft in Dresden).

8. II, S. 380.

9. AaO. S. 383.

10. Ebd. Anhang, S. 48.

11. Vgl. Krieg 1809, Bd. 1, hrsg. v. E. Mayerhoffer von Vedropolje, Wien 1907, S. 218, 237, 241. Hier wäre Sembdner zu berichtigen, dem zufolge die Landwehr "nur für den Schutz des Landes bestimmt war" (II, S. 930). - Noch am 25. Dezember 1808 hatte Erzherzog Karl für die Ausarbeitung der Kriegspläne die Anweisung erteilt, da $B$ die regulären Truppen zu offensiven Bewegungen außerhalb Österreichs zu verwenden seien, die Landwehr dagegen zur Verteidigung im Innerm; vgl. Manfried Rauchensteiner, Kaiser Franz und Erzherzog Carl. Dynastie und Heerwesen in Österreich 1796-1809, München 1972, S. 94.

12. Ferdinand Strobl von Ravelsberg, Die Landwehr Anno Neun, Wien u. Leipzig 1909, S. 69f; vgl. auch den Brief einer hochgestellten Persönlichkeit an Friedrich von Stadion vom 16. April 1808, der sich unter Hinweis auf das Landwehrpatent und den Zwischenfall in Böhmen gegen den Einsatz der Landwehr jenseits der Grenzen ausspricht und davor wamt, den guten Willen dieser Truppen aufs Spiel zu setzen (Haus-, Hof- und Staatsarchiv Wien, Kriegsakten 487, fol. 364-365). - Der Moniteur Universelle wies am 17. Mai 1809 auf die Schwierigkeiten mit der böhmischen Landwehr hin.

13. Die Landwehr gehörte zu den bevorzugten Zielen der napoleonischen Propaganda; siehe die entsprechenden Artikel im Moniteur (z.B. 4., 8., 14. April, 17., 19. Mai 1809) und im Journal de l'Empire (z.B. 20. März, 1., 11. April, 18. Juni 1809). In der am 12. Mai 1809 in München veröffentlichten Flugschrift Oesterreichische Proklamationen mit Anmerkungen heißt es: "Es ist bekannt, wie die Masse zur Bewaffnung getrieben wurde; es sind freiwillige Vaterland:svertheidiger, die man mit Stricken und Ketten vor sich her trieb." (S. 14).

14. Faksimile des Aufrufs in Strobl von Ravelsberg aaO. nach S. 70. - Diese Proklamation enthält übrigens Formulierungen, welche möglicherweise eine Anregung für die emphatische Schlußzeile des in jenen Tagen entstandenen Gedichts An den Erherzog Karl darstellen. Während Kleist den Erzherzog als "Überwinder des Unüberwindlichen" (I,S. 31) feiert, heißt es hier: "Kaiser Napoleon, der sich unüberwindlich dünkte, ist ... auf das Haupt geschlagen worden ... . Diese Armee ... hat den Nimbus seiner Unüberwindlichkeit zerstört ..."; vgl. hierzu auch vom Verf. "Heinrich von Kleists politisches Wirken" aaO. S. 29.

15. Sembdner Z.B. bemerkt zur Entstehung: "Nach der Schlacht von Regensburg (23. April 1809), aber vermutlich noch vor dem österr. Sieg bei Aspern (22. Mai 1809)." (II, S. 932).

16. II, S. 354.

17. Krieg 1809 , Bd. 1, aaO. S. $357 \mathrm{ff}$.

18. Vgl. I, S. 932f. - Wohlfeil untersucht die spanischen Vorlagen des Bürger-Katechismus (aaO. S. 247-251) und druckt einen spanischen Katechismus, den Bürger-Katechismus und Kleists Werk synoptisch ab (S. 309ff). Seine Ausführungen zu Form und Inhalt von Kleists Katechismus (S. 251-259) sind von der Kleistforschung noch nicht zur Kenntnis genommen worden.

19. Peter Rassow, "Die Wirkung der Erhebung Spaniens auf die deutsche Erhebung gegen Napoleon I." In: Historische Zeitschrift 167, 1942, S. 334.

20. Wohlfeil kommt $z u$ dem Schluß, daß Strauß wohl kaum politisch dachte, sondern die Flugschriften als Geschäftsmann vertrieb (aaO. S. 192). 21. Heinrich von Kleist's Participation aaO. S. $380,406$.

21. Heinrich von Kleist's Participation aaO. S. 380, 406. 
22. Krisenjahre der Frühromantik, 2. Aufl., hrsg. v. J. Kömer, Bd. 2, Bern u. München 1969 , S. 54f. Bereits am 15. Juni hatte Schlegel Joseph Hammer gegenüber seine Genugtuung über die Flucht von Strauß geäußert: "Ich möchte in Verbindung mit ihm bleiben. Vielleicht hätte er doch Gelegenheit nützlich zu sein, und wir könnten ihn in Thätigkeit setzen, da nun die Regierung sehr geneigt ist, mit der That auch durch das Wort zu erwidern." (Briefe von und an Friedrich und Dorothea Schlegel, hrsg. v. J. Körner, Berlin 1926, S. 117).

23. Zu F. Schlegels Wirken im Jahre 1809 sei hier aus österreichischen Akten Folgendes mitgeteilt: In einem in Znaim am 20. Mai 1809 verfaßten Schreiben des Armeeministers Zichy an Friedrich von Stadion heißt es über ihn, er sei "von Wien nach Brünn und von dort hierher gereiset, und benützet diese Gelegenheit, Eure Exzellenz um eine Bestimmung zu bitten, da er hier nicht verwendet werden kann." Auf dem Brief findet sich ferner der Vermerk "wird durch H. v. Schlegel überbracht" (Haus-, Hof- u. Staatsarchiv, Familienarchiv Stadion 109, fol. 341). - In einem Schreiben vom 27. Otober 1809 an Friedrich von Stadion, in dem Zichy die Auflösung des Oberhofkommissariats und der Provinzialkommissariate verfügt, bemerkt èr: "Der hofsekretär Schlegel bleibt einstweilen bei seinem Geschäfte und Genuße, und wird von mir hiervon unterrichtet." (ebd. 104/3, Nr. 5, fol. 46).

24. Haus-, Hof- u. Staatsarchiv Wien, Staatskanzlei Interiora, Korrespondenz 64, fol. 58.

25. Vgl. Buols Brief an Philipp von Stadion vom 24. April 1809 (Haus, Hof- u. Staatsarchiv Wien, Staatskanzlei Sachsen 38, fol. 180).

26. Vgl. Hellmuth Rößler, Osterreichs Kampf um Deutschlands Befreiung, Bd. 2, Hamburg 1940, S. 16. - Im Mai 1809 verhandelte der mit Kleist bekannte Karl von Nostitz mit den österreichischen Behörden über seinen Plan, mit einem Freikorps in Sachsen einzufallen; siehe Anton Ernstberger, Die deusschen Freikorps 1809 in Böhmen, Prag 1942, S. $311 \mathrm{ff}$.

27. Zum Aufenthalt Kleists in Znaim vgl. vom Verf. "Heinrich von Kleists politisches Wirken" aaO. S. 27.

28. Zur Datierung dieser Entwürfe vgl. ebd. S. 37. Hier sei noch nachgetragen, daß Anfang September 1809 als Entstehungsdatum auch insofern plausibel ist, als sich zu diesem Zeitpunkt die jeweils einmonatige Verlängerung des am 12. Juli 1809 in Znaim abgeschlossenen Waffenstillstandabkommens ihrem Ende näherte. - Zur Wirkung der Zensur auf die Presse in Böhmen siehe Heinrich von Kleists Lebensspuren. Dokumente und Berichte der Zeitgenossen, hrsg. v. H. Sembdner, Frankfurt 1977, Nr. 331.

29. II, S. 826. Zur Bestimmung des Empfängers vgl. vom Verf. "H. v. Kleists politisches Wirken" aaO. S. 27.

30. II, S. 377.

31. H. v. Kleists Lebensspuren aaO. Nr. 317-318.

32. II, S. 828. Das Wort "Aufsatz" hatte damals einen breiteren Anwendungsbereich als heute und konnte verschiedene literarische Formen bezeichnen; vgl. Vincent J. Dell'Orto, "Nineteenth-Century Descriptions of the German Essay." In: Jahrb. f. Internationale Germanistik 10, 1978, S. 8.

33. Heinrich von Kleist's politische Schriften, Berlin 1862, S. 10.

34. Vgl. z.B. Bethke aaO. S. 4, 85; Samuel aaO. S. 380; Berg aaO. S. 195.

35. Vgl. die folgenden Werke: Achtzehnhundertneun. Die politische Lyrik des Kriegsjahres, hrsg. v. Robert F. Arnold u. Karl Wagner, Wien 1909; Karl Wagner, "Die Flugschriftenliteratur des Krieges von 1809." In: Anno Neun. Volkslieder und Flugschriften, Brixen 1912, S. 63-155; Karl Wagner, "Die Wiener Zeitungen und Zeitschriften der Jahre 1808 und 1809." In: Archiv f. österr. Geschichte 104, 1915, S. 197-401. 